http://jmscr.igmpublication.org/home/ ISSN (e)-2347-176x ISSN (p) 2455-0450 crossref DOI: https://dx.doi.org/10.18535/jmscr/v8i2.43

\title{
Role of Diffusion-Weighted Magnetic Resonance Imaging of Brain in Patients with Neurological Deficits
}

\author{
Authors \\ Dr Thunga Swathi ${ }^{1}$, Dr Jayanti Musib ${ }^{2}$, Dr Julius N Toppo ${ }^{3}$, Dr Kada Venkataramana ${ }^{4}$ \\ ${ }^{1} 3$ rd year Post Graduate, ${ }^{2}$ 2nd year Post Graduate \\ ${ }^{3}$ Professor \& HOD, ${ }^{4}$ Professor
}

\begin{abstract}
Background and Objectives: Brain lesion is emerging as a distinct disease entity with significant morbidity. The disease characterized by sudden onset of neurological symptoms. The availability of diffusion-weighted magnetic resonance imaging (DW MRI) has become mandatory for accurate diagnosis as it provides information about the location, extent, and severity of the lesion.

The objectives of the present study are to define the brain lesions in clinically suspected neurological deficits patients with the help of diffusion-weighted magnetic resonance imaging.

Materials and Methods: The present cross-sectional study was conducted in the Department of Radiology, KIMS \& RF, Amalapuram, during the period of 2017 to 2019 among 40 clinically suspected patients with neurological deficits. The machine used in this study was PHILIPS achieva, 1.5 tesla MRI. For Diffusion-Weighted $b$ values used were 0 and $1000 \mathrm{~s} / \mathrm{mm}^{2}$, and the trace images were obtained by applying the gradients in three orthogonal directions.

Results: A maximum numbers of cases were males. The most frequent presenting complaints were neurological deficits. A maximum numbers of cases were of stroke lesions. All stroke lesions appear hyperintense on DWI and hypointense on ADC. All tumors ADC was high except epidermoid, pituitary adenoma, and GBM. All infectious lesions ADC was high except for two cases of encephalitis and one case of multiple tubercular abscesses.

Conclusion and Interpretation: DWI comes to an accurate diagnosis of stroke, tumors, infections, trauma, and demyelinating diseases.

Keywords: Diffusion-weighted Imaging, neurological deficits.
\end{abstract}

\section{Introduction}

The role of diffusion-weighted magnetic resonance imaging (DW MRI) in the evaluation of various intracranial lesions has been evolving and increasing since its initial introduction as newer uses explored always.

Though initially, DW MRI used for the early detection of stroke, now it has got various other clinical applications. DW MRI is a relatively recent imaging technique in which water selfdiffusion is a source of contrast on MR images. The molecular water proton diffusion process occurring on a micron-scale is imaged noninvasively and accurately without the use of any exogenous contrast. This measurement of water self-diffusion properties in the brain provides unique biological and clinically relevant information about the tissue composition, the 
physical structure of its constituents, tissue microstructure, and structural organization. It is currently not available from any other imaging modalities. However, this information is not yet fully exploited. The first measurements of water self diffusivity were reported in the earlier 1950s by Carr and Purcell ${ }^{[1]}$, whose conceptual framework formed the basis for modern diffusion imaging. Later in 1956, Torrey ${ }^{[2]}$ further worked on the diffusion of magnetization in Bloch (magnetization transport) equations. ${ }^{[3]}$ The analytical solutions to this equation followed for freely diffusing species during a spin-echo experiment ${ }^{[4]}$ and later for diffusion in restricted geometries. ${ }^{[5-7]}$

The next step in the evolution of DW MRI is the development and use of pulsed field gradients by Stejskal and Tanner ${ }^{[8]}$. They also developed the concepts of b-value and apparent diffusion coefficient (ADC) values. ${ }^{[9]}$ However, all these are spin-echo techniques and are time-consuming and thus result in motion artifacts. The increased use of DW MRI is possible because many of the newer MR units are echo-planar capable. Echoplanar DW MRI is obtained within a few seconds and so is much less sensitive to motion artifacts than other imaging techniques. Role of DW MRI in stroke: As cytotoxic edema occurs in just a few minutes of a critical decrease in cerebral blood flow, DWI may show most acute infarctions. It is particularly useful when thrombolytic therapy, which can only be used within a short window of opportunity, is considered. Also, DWI identifies acute lesions in patients with multiple chronic ischemic lesions. It also identifies the new extension of the previous ischemic lesions. In addition to its role in the evaluation of acute cerebral ischemia, new uses for diffusionweighted imaging are:

1. In differentiating Arachnoid cysts versus Epidermoid

2. Primary and metastatic brain tumoursADC values may be used to grade the tumors.

3. Characterization of Ring enhancing lesions (abscess versus necrotic tumor)

4. Demyelinating diseases.

5. Head injury.

6. Evaluation of intracranial infections.

Brain lesions emerging as a distinct disease entity with significant morbidity. The disease characterized by sudden onset of neurological symptoms. With its availability, diffusionweighted magnetic resonance imaging (DW MRI) has become mandatory for accurate diagnosis as it provides information about the location, extent, and severity of the lesion. Diffusion-weighted (DW) magnetic resonance (MR) imaging provides image contrast that is different from that provided by conventional MR techniques. It is sensitive for the detection of acute ischemic stroke and its differentiation from other processes that manifest with sudden neurologic deficits. Diffusionweighted MR imaging also provides information for other cerebral diseases like neoplasms, intracranial infections, traumatic brain injury, and demyelination, stroke being common. In the most differential diagnosis of acute neurologic events, diffusion-weighted MR imaging should be considered an essential sequence. ${ }^{[10]}$ Its use is recommended in most brain MR studies. Patients of multiple sclerosis have increased water diffusion in the thalamus that is partly associated with lesion load, clinical course, and brain atrophy. Both direct and indirect mechanisms of gray matter injury may play a role in the pathophysiology of multiple sclerosis. ${ }^{[11]}$ Hence this study is proposed to analyze the pattern of various brain lesions with the help of DWMRI.

\section{Aims \& Objectives}

The present study is aimed to define the brain lesions in clinically suspected neurological deficits in patients using diffusion-weighted magnetic resonance imaging.

\section{Materials \& Methods}

This present study was done in the Department of Radiology, Konaseema Institute of Medical Sciences \& RF, Amalapuram, from 2017 to 2019. 
Study Design: Institution-based cross-sectional study.

Source of Data : All clinically suspected patients with neurological deficit (signs and symptoms includes dysphasia/aphasia, hemiparalysis, hemiparesis, global aphasia, cortical blindness, pinpoint pupil, severe headache, projectile vomiting, convulsions, papilloedema, vertigo, ataxia) referred by consultant neurologist, consultant neurosurgeon, consultant physician for DW MRI in Konaseema Institute Of Medical Sciences \& RF over a period of two years. The data is collected from patients undergoing MRI.

Sample Size: 40 patients with neurological deficits.

Sample Size calculation: $80 \%$ Average number of cases admitted to Konaseema Institute Of Medical Sciences \& RF, Amalapuram in the previous three years.

\section{Selection Criteria:}

\section{Inclusion Criteria}

All cases referred for DW MRI with clinically suspected sudden neurological deficits attending Konaseema Institute Of Medical Sciences \& RF, Amalapuram over two years.

\section{Exclusion Criteria}

All patients with cardiac pacemakers, prosthetic heart valves, any metallic orthopedic implants, and patients on artificial respiration.

\section{Procedure}

\section{Data Collection}

All the patients with neurological deficits referred by a consultant neurologist, consultant neurosurgeon, consultant physician for DW MRI in KIMS \& RF were screened for eligibility. The eligible patients were administered informed consent. The consented participants were enrolled in the present study. A complete clinical history of the patients was recorded on predesigned and pretested proforma, which included, age sex, principal presenting complaints.

Equipment: The machine used in this study was PHILIPS achieva, 1.5 tesla MRI.

\section{Mri Protocol}

After the general examination, the patient was taken for MRI, and axial T1 weighted, axial T2 weighted, FLAIR and diffusion-weighted images were obtained.

For Diffusion-Weighted, $\mathrm{b}$ values used were 0 and $1000 \mathrm{~s} / \mathrm{mm} 2$, and trace images were obtained by applying the gradients in three orthogonal directions. A $5 \mathrm{~mm}$ thick slice with a spacing of 1 $\mathrm{mm}$ was used over an acquisition time of $40 \mathrm{~s}$.

Contrast studies were performed whenever requested by the clinician and were also performed when tumors and infection were suspected of initial diagnosis.

The lesions were characterized based on their intensity into hypointense, isointense, and hyperintense on diffusion-weighted and apparent diffusion coefficient (ADC).

\section{Observations \& Results}

\section{Sex distribution}

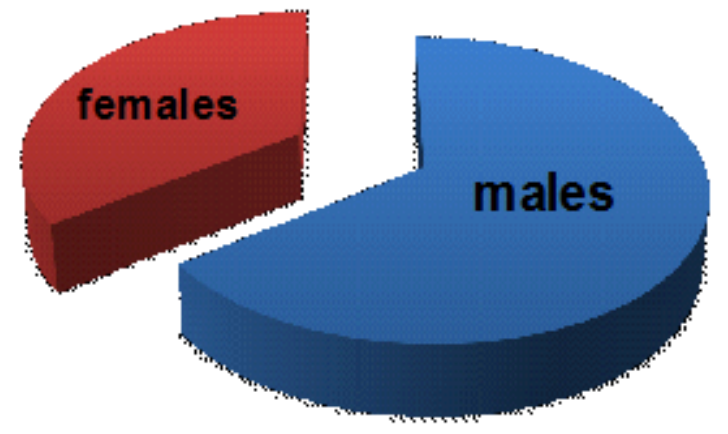

In the present study, the male population predominates, 26 patients $(65 \%)$, the female population being 14 patients $(35 \%)$. The male: female ratio is $1.85: 1$. 


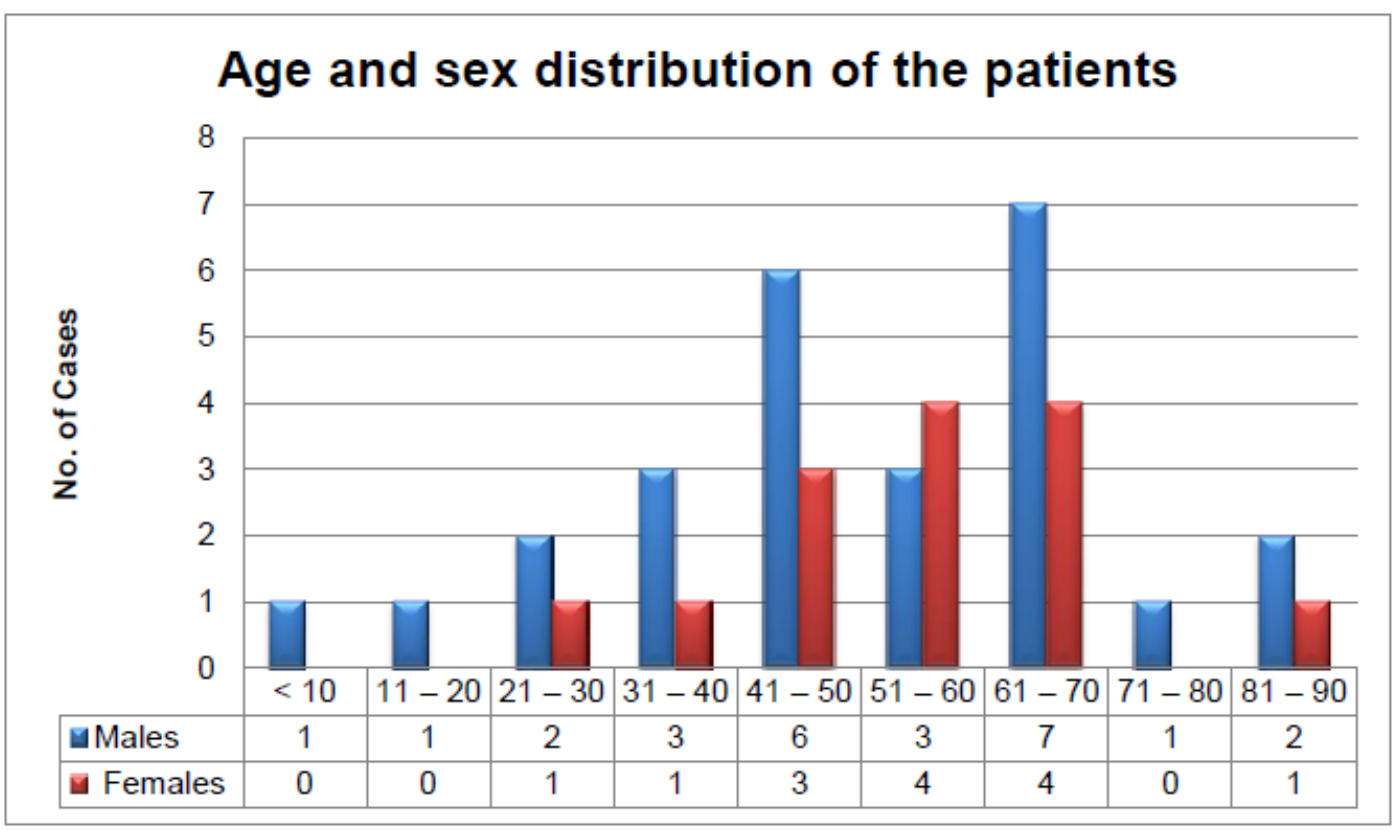

In the present study, the peak incidence of neurological deficits in males occurred in the age group of 61-70 that is seven patients (17.5\%)
In females, the peak incidence occurred in $51-60$ and $61-70$ age groups that are four patients each $(10 \%)$.

Distribution of patients according to MR diagnosis

\section{Distribution of patients according to MR diagnosis}

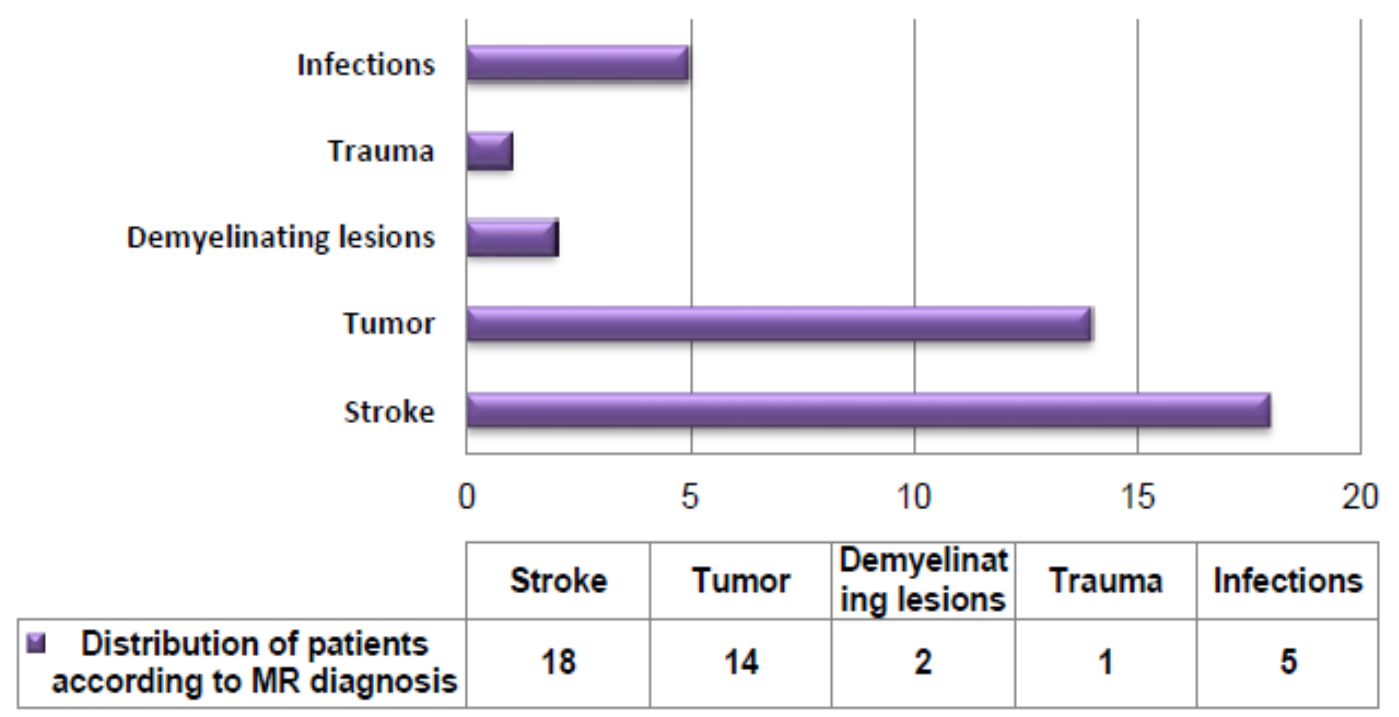

Distribution of stroke patients with /without hemorrhagic transformation

\begin{tabular}{|l|c|c|}
\hline \multicolumn{1}{|c|}{ Diagnosis } & Number & Percentage \\
\hline Acute ischemic lesions without hematoma / HT & 15 & $83.33 \%$ \\
\hline Acute stroke: Lesions with hematoma / HT & 03 & $16.66 \%$ \\
\hline Total & 18 & $100.00 \%$ \\
\hline
\end{tabular}


Cases of stroke detected by DW MRI with T2W MRI techniques

\begin{tabular}{|l|c|}
\hline \multicolumn{1}{|c|}{ Diagnostic technique } & No. of patients \\
\hline Lesions are seen only on OW MR & 03 \\
\hline Lesions are seen both on OW MR and T2WI & 14 \\
\hline Multiple lesions are seen on T2, and one is prominent on DWI & 01 \\
\hline
\end{tabular}

In the present study, the majority of the lesions were seen (Fourteen patients) on both DW MR and $\mathrm{T} 2$, followed by three patients seen only on
DW MR and one patient with multiple lesion on T2 and prominent of DWI.

Summary of patients tested for stroke classified based on time of symptoms have occurred

\begin{tabular}{|l|c|c|c|}
\hline Duration of symptoms & No. of patients & $\begin{array}{c}\text { DW MR } \\
\text { sensitivity (\%) }\end{array}$ & $\begin{array}{c}\text { T2-W MRI } \\
\text { sensitivity \% }\end{array}$ \\
\hline Within 3 hours & 04 & $100.00 \%$ & $25.00 \%$ \\
\hline $3-6$ hours & 04 & $100.00 \%$ & $75.00 \%$ \\
\hline $6-24$ hours & 05 & $100.00 \%$ & $100.00 \%$ \\
\hline $24-48$ hours & 03 & $100.00 \%$ & $100.00 \%$ \\
\hline $2-7$ days & 02 & $100.00 \%$ & $100.00 \%$ \\
\hline
\end{tabular}

The difference in sensitivity gradually decreases as time progresses.

\section{Incidence of different types of Tumors}

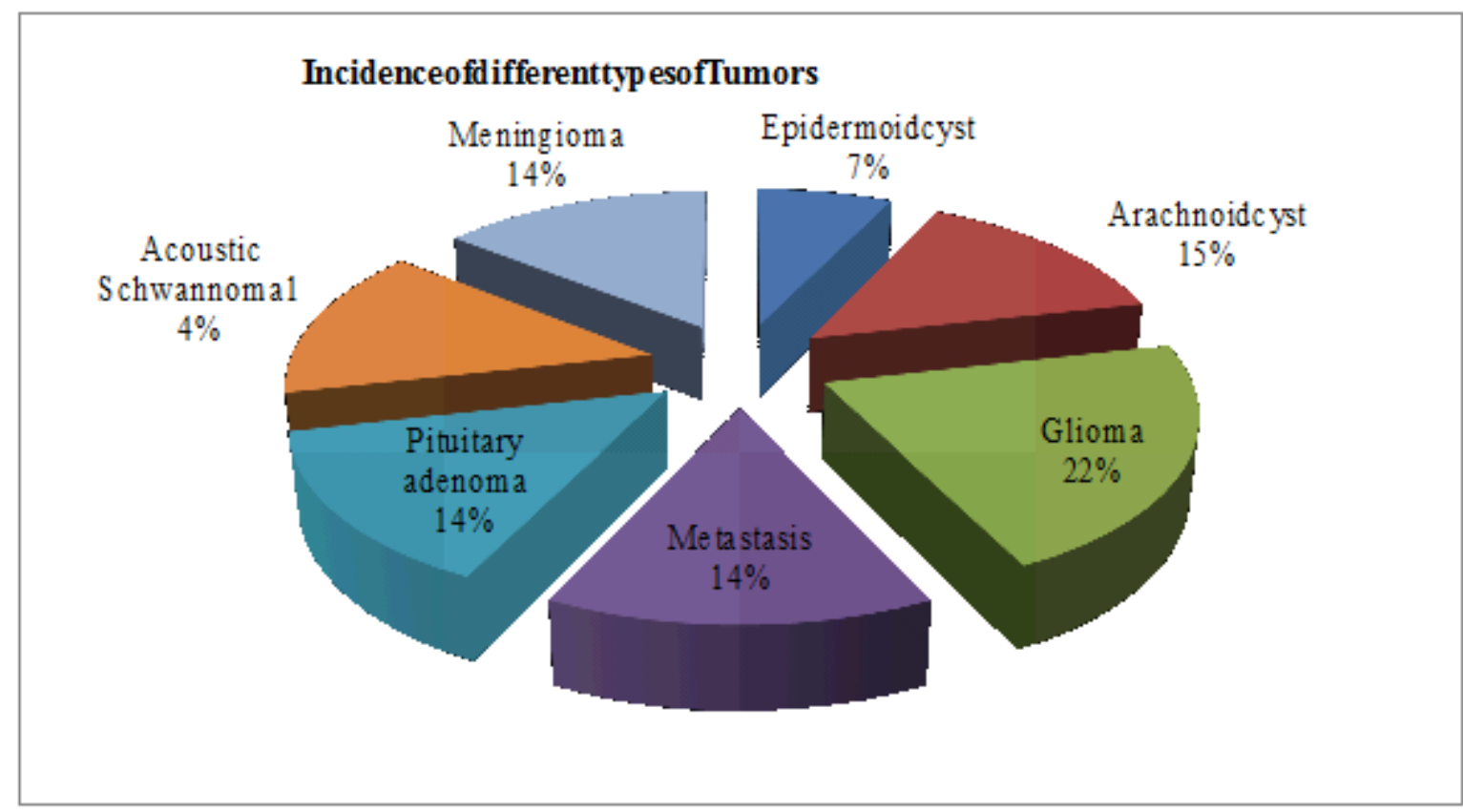

Morphology of lesions in stroke on DWI \& ADC

\begin{tabular}{|l|c|c|c|}
\hline Stroke & $\begin{array}{c}\text { Number of } \\
\text { patients }\end{array}$ & DWI & ADC \\
\hline $\begin{array}{l}\text { Acute } \\
\text { hemorrhagic/hematoma conversion }\end{array}$ & 15 & Hyperintense & Hypointense \\
\hline $\begin{array}{l}\text { Acute ischemic lesions with } \\
\text { hematoma/hemorrhagic conversion }\end{array}$ & 03 & Hyperintense & Hypointense \\
\hline
\end{tabular}

All stroke lesions appear hyperintense on DWI and hypointense on ADC. 
Morphology of tumors on ADC

\begin{tabular}{|l|c|c|}
\hline \multicolumn{1}{|c|}{ Tumors } & No.of cases & ADC \\
\hline Epidermoid cyst & 01 & HYP \\
\hline Arachnoid cyst & 02 & HI \\
\hline Glioma & 03 & HI(2), HYP(1) \\
\hline Meningioma & 02 & ISO/HI \\
\hline Metastasis & 02 & HI \\
\hline Pituitary adenoma & 02 & HYP \\
\hline Acoustic Schwannoma & 02 & HI \\
\hline
\end{tabular}

Tumors ADC was high in all except epidermoid, pituitary adenoma, and GBM

Tumors \& its distribution according to age

\begin{tabular}{|l|c|c|c|c|c|c|c|c|}
\hline Age in Years & \multicolumn{9}{|c|}{ Tumors } \\
\cline { 2 - 9 } & $\begin{array}{c}\text { Epiderm } \\
\text { oid cyst }\end{array}$ & $\begin{array}{c}\text { Arachnoid } \\
\text { cyst }\end{array}$ & $\begin{array}{c}\text { Glio } \\
\text { ma }\end{array}$ & $\begin{array}{c}\text { GBM } \\
\text { Menin } \\
\text { gioma }\end{array}$ & $\begin{array}{c}\text { Metast } \\
\text { asis }\end{array}$ & $\begin{array}{c}\text { Pituitary } \\
\text { adenoma }\end{array}$ & $\begin{array}{c}\text { Acoustic } \\
\text { Schwannoma }\end{array}$ \\
\hline$<10$ & 00 & 01 & 00 & 00 & 00 & 00 & 00 & 00 \\
\hline $11-20$ & 00 & 01 & 00 & 00 & 00 & 00 & 00 & 00 \\
\hline $21-30$ & 01 & 00 & 00 & 00 & 00 & 00 & 00 & 01 \\
\hline $31-40$ & 00 & 00 & 00 & 00 & 00 & 00 & 00 & 01 \\
\hline $41-50$ & 00 & 00 & 00 & 00 & 01 & 00 & 00 & 00 \\
\hline $51-60$ & 00 & 00 & 01 & 00 & 01 & 01 & 00 & 00 \\
\hline $61-70$ & 00 & 00 & 01 & 00 & 00 & 00 & 00 & 00 \\
\hline $71-80$ & 00 & 00 & 00 & 00 & 00 & 01 & 02 & 00 \\
\hline $81-90$ & 00 & 00 & 00 & 01 & 00 & 00 & 00 & 00 \\
\hline Total & 01 & 02 & 02 & 01 & 02 & 02 & 02 & 02 \\
\hline
\end{tabular}

In the present study, two patients each had metastasis in the age group of 51 to 60 years, and 71 to 80 years, two had Glioma, one in 51 to 60 years, one in 61 to 70 years, and one had GBM in 71 to 80 years. Two patients had pituitary adenoma in the age group of 61 to 70 years. Two patients had arachnoid cysts, one each in age groups of $<10$ years and 11 to 20 years. Two patients had Acoustic schwannoma, one in the age group 21 to 30 years, and one in 31 to 40 years. Two patients had meningiomas, one each in age groups of 41 to 50 years and 51 to 60 years. One patient had an epidermoid cyst in the age group of 21 to 30 years.

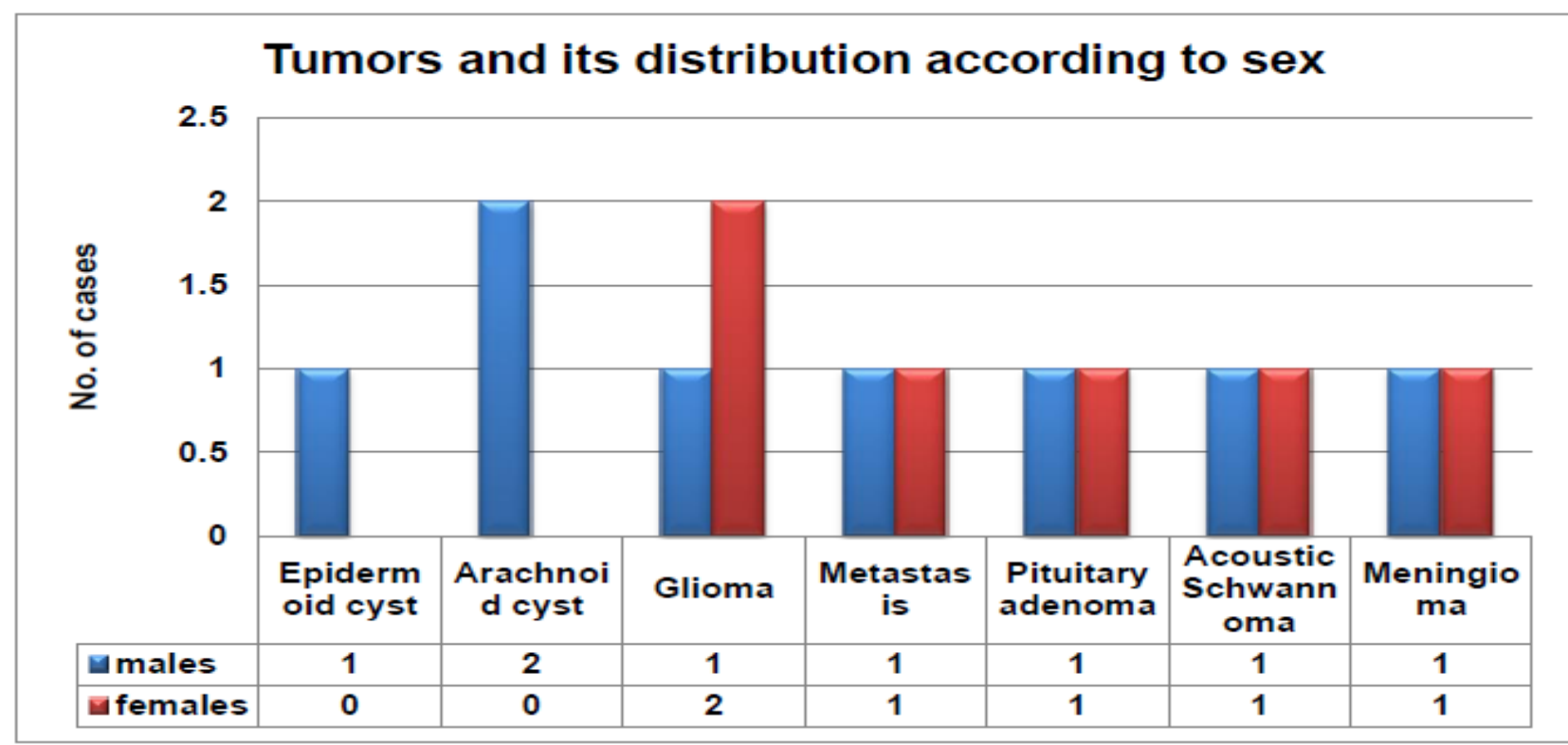

In the present study of tumors, the male population predominates, i.e., eight patients

$(57.14 \%)$ and six female patients $(42.85 \%)$. The male: female ratio is $4: 3$. 


\section{Discussion}

In the present study out of 40 patients, eighteen patients $(45 \%)$ had stroke, $14(35 \%)$ had tumors, five patients $(12.5 \%)$ had infections, two patients $(5 \%)$ had demyelinating lesions, and one patient $(2.5 \%)$ had trauma.

\section{Stroke}

Of 18 stroke patients, acute ischaemic lesions without hemorrhagic/ hematoma transformation were more that is $83 \%$ (15 patients), and lesions with hematoma /hemorrhagic transformation were of less number that is $17 \%$ (3 patients). In the present study of 18 stroke patients, a total of 3 patients $(16.66 \%)$ with HT were studied. The sensitivity of DW imaging in identifying hematomas is $77.77 \%$, and specificity is $100 \%$. We did not use gradient-echo as a standard for comparison. We only used $\mathrm{T} 1$ and $\mathrm{T} 2 \mathrm{~W}$ images for identifying and characterizing hematomas. The results were not consistent with the results of a similar study ${ }^{[12]}$

In the present study, of 18 stroke patients, four patients presented within 3 hours, and four patients presented within 3-6 hours of symptoms onset. The sensitivity of DWI was $100 \%$ in both the periods, with the sensitivity of T2WI being $25 \%$ and $75 \%$, respectively. The results were similar to a study ${ }^{[13]}$ study done on 53 patients (mean age, $66 \pm 14$ years) with suspected recent cerebral ischemia. In 39 patients (74\%) DWI clearly delineated recent ischemic damage as compared with $33(62 \%)$ in whom lesions were identified on conventional T2-weighted images and concluded that in DWI sequences, recent ischemic damage is better shown than on conventional and contrast- enhanced MR images within first days after stroke and may provide information about the origin of clinical symptoms. Adding DWI to imaging protocols for patients with subacute cerebral ischemia is recommended.

\section{Primary and Secondary Brain tumors}

In a study done by Comelli I et al ${ }^{[14]}$ on clinical presentation and epidemiology of brain tumors, a retrospective study in 2017, gliomas were more common(46.3\%), next comes meningiomas (22\%), metastasis (17\%) and miscellaneous $(30 \%)$. The present study showed a higher incidence $(22 \%)$ of gliomas next comes others, meningiomas (14\%), pituitary adenoma(14\%) and metastasis(14\%), acoustic schwannomas and arachnoid cysts each $14 \%$, epidermoid cyst $(7 \%)$ when correlated with the above mentioned studies.

\section{Demyelinating diseases}

In two patients with demyelination, the ADC values within the lesion were low. The number of lesions identified on DW imaging was more compared to T2 WI. The present study correlated with the case study ${ }^{[15]}$ as restricted diffusion was noted when the study was done within four days. In the present study, diffusion restriction was seen in both the patients, but they were not ringenhancing lesions as compared to another study, ${ }^{[16]}$

\section{Trauma}

In the present study, one patient who presented within 2 hours of RTA had no positive findings of CT imaging followed by MRI the next day. Patient had DAI with T2/FLAIR HI areas noted with foci of blooming on GRE and showed restricted diffusion which correlated with another study ${ }^{[17]}$, they concluded that DWI is valuable in closed head injury as it identifies additional shearing injuries not visible on T2/FLAIR or T2* sequences.

\section{Infections}

In a study ${ }^{[18]}$, they performed MRI including T1W, T2-W/ FLAIR sequences, and DWI in 18 patients in whom encephalitis was diagnosed, there was an excellent correlation between ADC values and duration of the disease they concluded that in the early viral encephalitic stages the detection of lesions and depiction of the lesion borders, DWI in combination with other sequences, may contribute to the determination of the disease phase and is superior to other conventional sequences results in the present 


\section{JMSCR VoI||08||Issue||02||Page 226-235||February}

study also correlated with three encephalitis patients, one patient showed high ADC values, and the other two patients showed restricted diffusion with low ADC values.
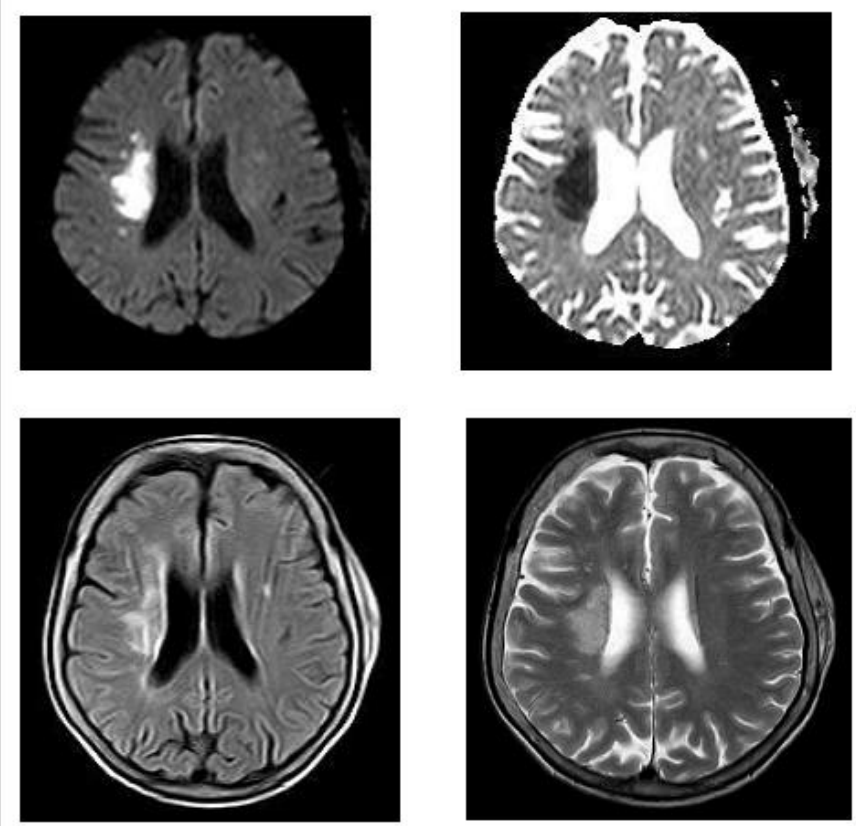

Acute R MCA territory infarct

Restricted diffusion noted in the right perisylvianfrontoparietal cortex \& white matter.
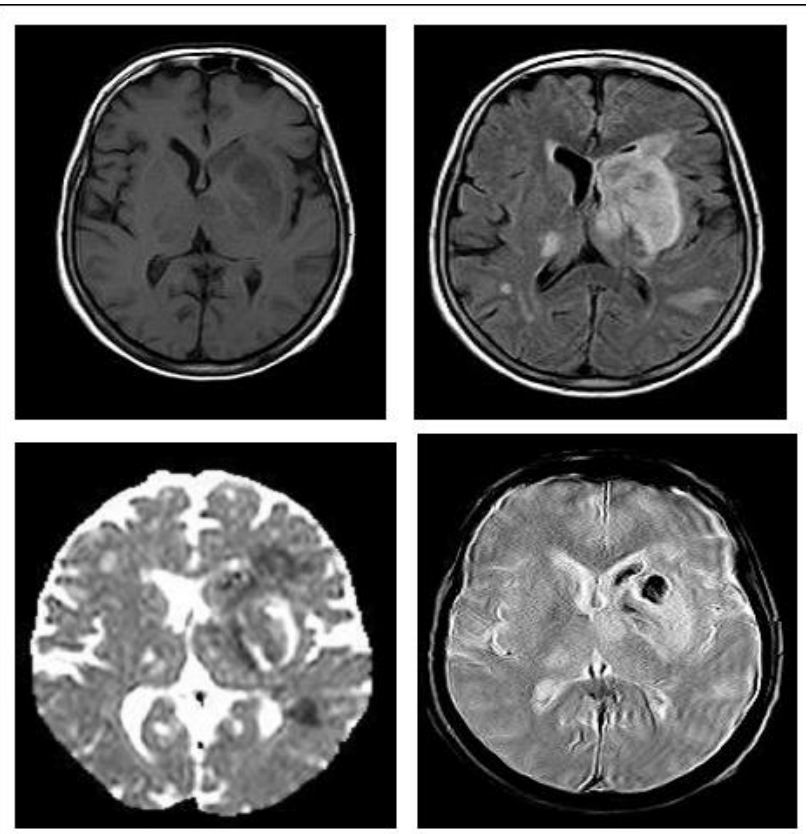

Acute Infarct with HT involving left MCA territory

T2/FLAIR HI noted with blooming foci on GRE and with mass effect.
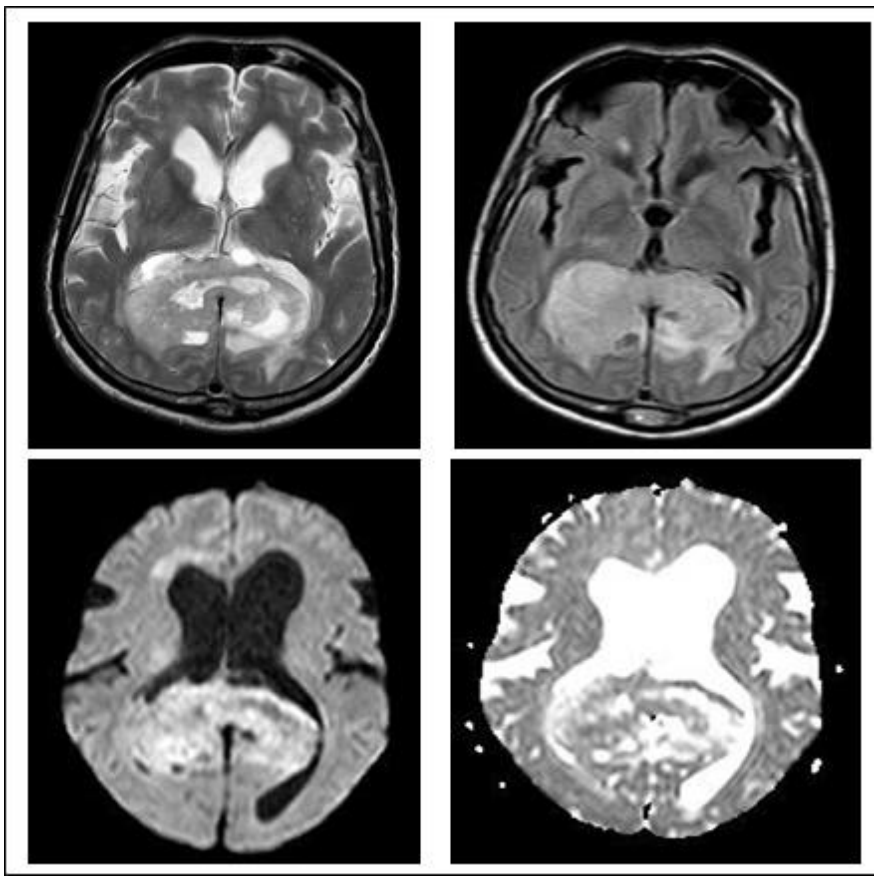

85-Year-Old Female with Glioblastoma

Two epi.of seizures, visual disturbances.

Enhancing mass lesion with blooming foci and perifocal edema in splenium of $\mathrm{CC}$ with Restricted diffusion.
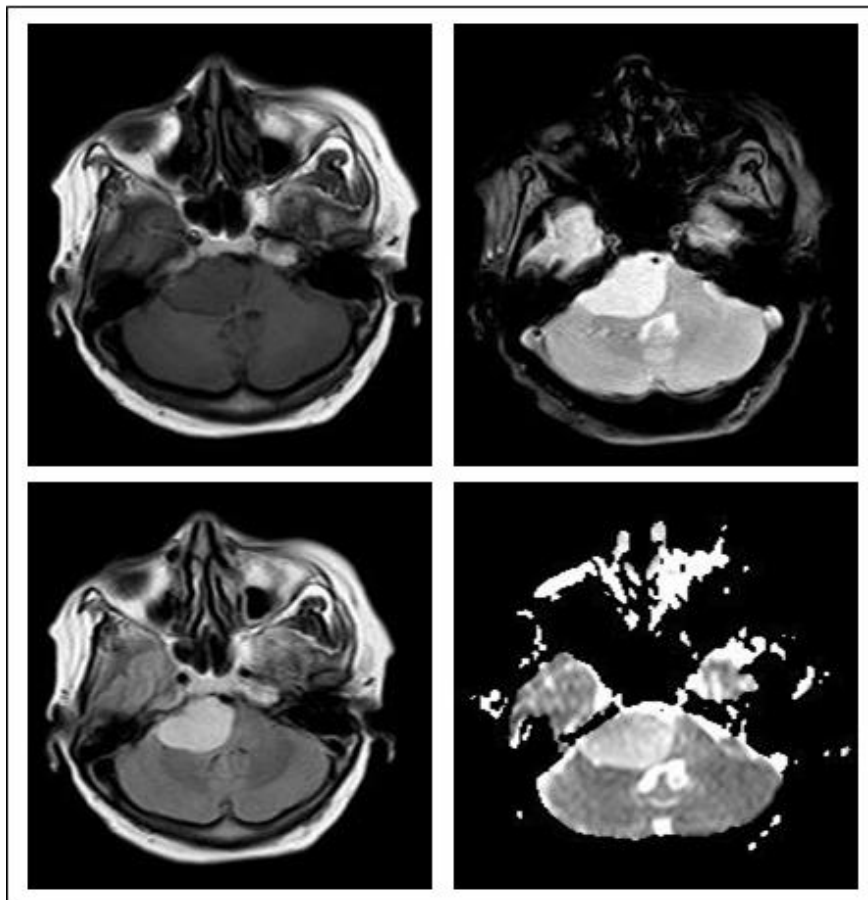

Meningioma in Right CP Angle

$50 \mathrm{y} / \mathrm{F}$, Right eye pain radiating to $\mathrm{rt}$ ear.

Extra axial T2/FLAIR HI lesion in rt $\mathrm{CP}$ angle.

On ADC- iso/ hyper intense 


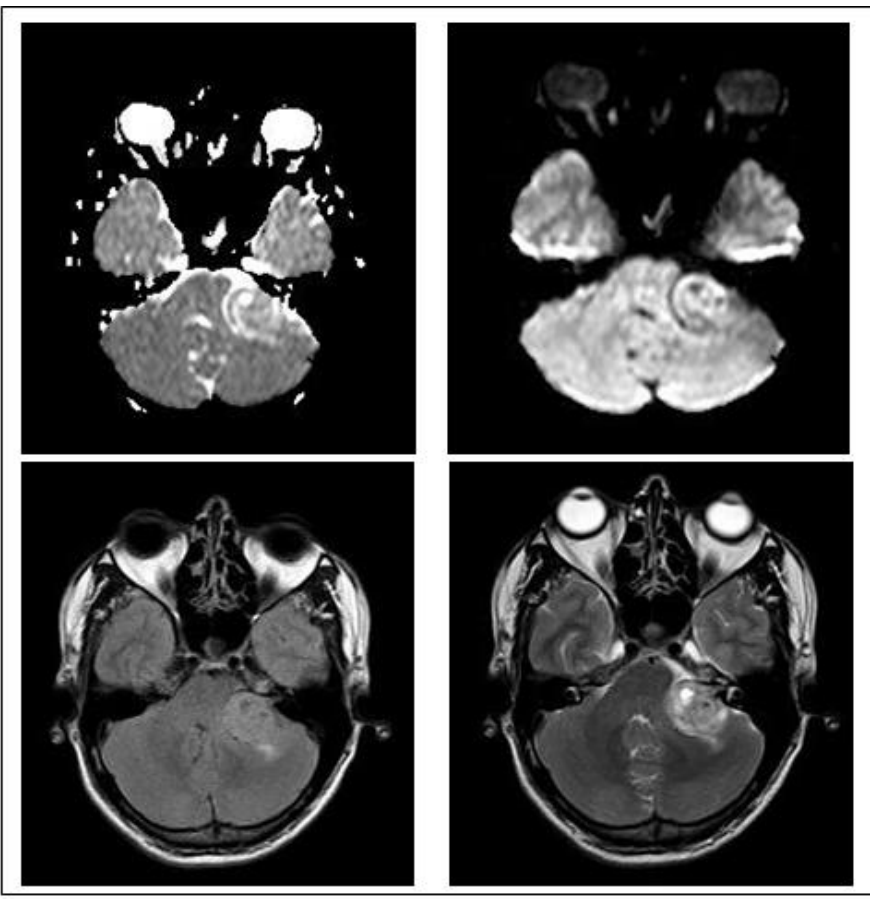

Acoustic schwannoma

$24 \mathrm{y} / \mathrm{F}$, hearing loss, headache, ringing in ear.

T2/FLAIR Heterogeneous signal intensity extraaxial mass lesion in the left $\mathrm{CP}$ angle. Hyperintense on ADC. Mass effect on brachium pontis noted.

\section{Conclusion}

The DW MR is a very useful sequence in the first 6 hours of the onset of stroke- like symptoms. DW MR cannot identify hyperacute bleeds detected on conventional sequences. In patients with multiple infarcts, DW MR identifies the acute lesion. DW MR is beneficial in differentiating epidermoids and arachnoids. There is considerable overlap in the ADC values of low grade and high- grade tumors. So, we cannot reliably grade tumors based on ADC values, but we may guide a very cellular area for stereotactic biopsy. Also, tumor margins are not delineated on DW MR compared to T1 and $\mathrm{T} 2 \mathrm{~W}$ imaging. In demyelinating disease, a comparative number of lesions were identified on DW MR, and the ADC values of these lesions are variable. In trauma, with diffuse axonal injury, DW MR identified more lesions, and the ADC values are persistently low of trauma, suggesting the possibility of neurotoxic edema. In infections, DW MR, due to its poor resolution, DW MR could not identify some of the small lesions seen on conventional MR sequences. We can characterize the type of edema on DW MR, and if there is cytotoxic edema, the possibility of underlying vasculitis can be contemplated.

\section{References}

1. Carr HY, Purcell EM. Effects of diffusion on free precession in nuclear magnetic resonance experiments. Physical review. 1954 May 1;94(3):630.

2. Torrey HC. Bloch equations with diffusion terms. Physical review. 1956 Nov 1;104(3):563.

3. Bloch F. Nuclear induction. Physical review. 1946 Oct 1;70(7-8):460.

4. Douglass DC, McCall DW. Diffusion in paraffin hydrocarbons. The Journal of Physical Chemistry. 1958 Sep;62(9):11027.

5. Neuman $\mathrm{CH}$. Spin echo of spins diffusing in a bounded medium. The Journal of Chemical Physics. 1974 Jun 1;60(11):4508-11.

6. Stejskal EO. Use of spin echoes in a pulsed magnetic- field gradient to study anisotropic, restricted diffusion, and flow. The Journal of Chemical Physics. 1965 Nov 15;43(10):3597-603.

7. Wayne RC, Cotts RM. Nuclear-magneticresonance study of self-diffusion in a bounded medium. Physical Review. 1966 Nov 4;151(1):264.

8. Stejskal EO, Tanner JE. Spin diffusion measurements: spin echoes in the presence of a time- dependent field gradient. The journal of chemical physics. 1965 Jan 1;42(1):288-92.

9. Tanner JE. Transient diffusion in a system partitioned by permeable barriers. Application to NMR measurements with a pulsed field gradient. The Journal of Chemical Physics. 1978 Aug 15;69(4): 1748-54.

10. Ar Un's MRI Protocols: MRI DIFFUSION. 
https://mriprotocol.blogspot.com/2011/11/ mri-diffusion.html

11. Fabiano AJ, Sharma J, WeinstockGuttman B, Munschauer Iii FE, Benedict RH, Zivadinov R Bakshi R. Thalamic involvementin multiple sclerosis: a diffusion- weighted magnetic resonance imaging study. Journal of Neuroimaging 2003 Oct;13(4):307-14.

12. Atlas SW. Diffusion measurements in intracranial hematomas: Implications for MR imaging in acute stroke. AJNR 2000; 21: 1190-4.

13. Augustin M, Bammer R, Simbrunner J, Stollberger R, Hartung HP, Fazekas F. Diffusion-weighted imaging of patients with subacute cerebral ischemia: comparison with conventional and contrast-enhanced MR imaging. American journal of neuroradiology. 2000 Oct 1;21(9):1596-602.

14. Comelli I, Lippi G, Campana V, Servadei F, Cervellin G. Clinical presentation and epidemiology of brain tumors firstly diagnosed in adults in the Emergency Department: a 10-year, single center retrospective study. Annals of translational medicine. 2017 Jul;5(13).

15. Bernarding J. Diffusion- and perfusionweighted MR imaging in a patient with acute demyelinating encephalomyelitis (ADEM), J Magn Res Img 2002; 15: 96100.

16. Koelblinger C, Fruehwald-Pallamar J, Kubin K, Wallner-Blazek M, Van den Hauwe L, Macedo L, Puchner SB, Thurnher MM. Atypical idiopathic inflammatory demyelinating lesions (IIDL): Conventional and diffusionweighted MR imaging (DWI) findings in 42 cases. European journal of radiology. 2013 Nov 1;82(11):1996-2004.
17. Huisman TA, Sorensen AG, Hergan K, Gonzalez RG, Schaefer PW. Diffusionweighted imaging for the evaluation of diffuse axonal injury in closed head injury. Journal of computer assisted tomography. 2003 Jan 1;27(1):5-11.

18. Kiroğlu Y, Calli C, Yunten N, Kitis O, Kocaman A, Karabulut N, Isaev H, Yagci B. Diffusion-weighted MR imaging of viral encephalitis. Neuroradiology. 2006 Dec 1;48(12):875-80. 